\title{
Coping strategies, stigmatizing attitude, and cyberbullying among Chinese college students during the COVID-19 lockdown
}

\author{
Fan Yang ${ }^{1,2,3}$. Jian Sun ${ }^{1}$ Jiaying $\mathrm{Li}^{1}$ · Shoujun $\mathrm{Lyu}^{1,2,3}$ \\ Accepted: 2 February 2022 \\ (c) The Author(s), under exclusive licence to Springer Science+Business Media, LLC, part of Springer Nature 2022
}

\begin{abstract}
The ongoing COVID-19 pandemic provides hotbed for hatred and violence, which could be especially true among college students, the most active users of internet and social media. Based on a national sample of Chinese college students $(N=1,673)$, the present study aims to explore the clustered nature of stress coping strategies, as well as its associations with the participants' stigmatizing attitude and cyberbullying behaviors towards people in Hubei Province, the place where the first COVID-19 case was reported and recognized as China's epicenter of the pandemic. Four latent subgroups were first identified among the participants based on type and comparative adoption rate of their coping strategies, namely the emotional coping group, the inactive coping group, the support-seeking and positive coping group, and the independent and positive coping group. The significant associations between coping strategy patterns and stigmatizing attitude and cyberbullying behaviors were reported, respectively. The two were most likely to happen among the participants using emotional coping while the least likely among the independent and positive coping group. This study provides empirical supports for combating the secondary disasters of the pandemic, namely stigma and cyberbullying, by identifying the role of emotional and positive coping strategies.
\end{abstract}

Keywords COVID-19 $\cdot$ Stigmatizing attitude $\cdot$ Cyberbullying $\cdot$ Coping strategies $\cdot$ College students

\section{Introduction}

Students in higher education are faced with increasing risks of hatred and violence during the COVID-19 pandemic, especially stigmatizing and cyberbullying (Alsawalqa, 2021; Kim \& Choi, 2021). The major reason could be the more reliance on electronic devices and social media under the implementation of social distancing rules and online courses (Chiolero, 2020). Hatred and violence are closely associated with loneliness, fear, hopelessness, and self-esteem

Shoujun Lyu

sjlv@sjtu.edu.cn

Fan Yang

fan_yang86@sjtu.edu.cn

1 School of International and Public Affairs, Shanghai Jiao Tong University, Room 335, Xinjian building, 1954 Huashan Road, Shanghai, China

2 China Institute for Urban Governance, Shanghai Jiao Tong University, Shanghai, China

3 Institute of Health Yangtze River Delta, Shanghai Jiao Tong University, Shanghai, China reduction, which could further increase the risk of behavioral and emotional problems, such as alcohol misuse, depression, and suicide (Holmes et al., 2020; Yang, 2021).

College students and other young people could experience multiple stressors caused by the COVID-19, including fear of exposure, widespread rumors, and reduced job opportunities (Altena et al., 2020; Qiu et al., 2020). According to McCauley et al. (2013), people have soothed their stress of disease outbreaks by stigmatizing certain social groups throughout the history. In a similar vein, people living in Hubei, China's epicenter of the COVID-19 pandemic, are faced with high risks of stigmatization (Goh, 2020). Since late February of 2020, when the pandemic had spread for over two months and reached the peak in China, the whole country was strictly locked down and the resulting inconveniences and losses started to drive people crazy. Fear of the virus and suspicious people prevailed, and the social mood was fueled and distorted by tons of rumors about Hubei people and Hubei government's misconducts in dealing with the pandemic (Yang \& Huang, 2021). As a result, hating and stigmatizing any people or things that had connection with Hubei Province became popular. Because of the lock-down 
policy, people's hatred and stigmatization attitude towards Hubei people were conveyed mainly through internet.

And in the digital age, especially when lockdown is implemented, stigmatization and cyberbullying behaviors could easily run rampant. At the same time, people adopt various strategies to cope with the pandemic-related life stressors. These coping strategies may affect people's aggressive behaviors, especially those of young people (Bodenmann et al., 2010; DiClemente \& Richards, 2019). Therefore, exploring coping strategies may have implications for reducing the hatred and violence associated with the pandemic.

\section{Hatred and Violence in Public Health Crisis}

The past literature has well documented the increase of hatred and violence in public health crisis. Especially, the wide application of social media makes stigmatizing attitudes and cyberbullying behaviors more prevalent in the current COVID-19 pandemic (Wong et al., 2020). Two major forms of stigmatization during the public health crisis period were reported, including (1) stigmatizing people infected with certain disease (Garaigordobil \& Larrain, 2020); and (2) stigmatizing people with certain demographic characteristics that relate with that disease, which is now best represented by people in Hubei, the place where the first COVID-19 case was reported (Goh, 2020). At the same time, according to Mishna et al. (2020), cyberbullying is largely a result of perceived difference and is bias or stigmabased. And the intersectionality of different types of stigmatization may exacerbate cyberbullying (Lauckner et al., 2019; Mishna et al., 2020).

During the COVID-19 pandemic when unprecedented digital interconnectedness and intense media coverage have amplified the pandemic-associated psychological fear, college students had to stay indoors and move their classes online for prolonged periods of time (Chiolero, 2020), which could significantly influence their social network and make them more mentally vulnerable (Altena et al., 2020). During this period, they could use electronic devices excessively, be more easily affected by online rumors and biased news, and vent their negative emotions online (Wong et al., 2020). Though it is obvious that the COVID-19 provides hotbed for stigmatizing attitude and cyberbullying behaviors, still little is known about their prevalence among college students during the lockdown.

\section{Coping Strategy and Its Clustered Nature}

Taylor and Stanton (2007) defined coping strategy as "conscious volitional efforts to regulate emotion, thought, behavior, physiology and the environment in response to stressful events or circumstances". Fluharty and Fancourt (2021) found that people in UK would adopt varied coping strategies during COVID-19 pandemic, according to the availability of resources used to deal with a stressor. That is, persons with sufficient resources may select a positive-engaged coping strategy, such as finding a good balance between emotional expression and emotional regulation, or cognitive restructuring; while those with insufficient resources may adopt a negative-engaged coping strategy, such as avoidance or antagonizing people who seem threatening.

Other researchers revealed different factor structures of coping strategy. For instance, Endler et al. (1998) found that people may choose from three types of coping strategies, including problem-focused coping, emotion-focused coping, and avoidant coping, when faced with health problems. Moreover, based on a French sample, Doron et al. (2014) put forward a five-factor coping strategy model, namely problem-solving, support-seeking, avoidance, cognitive restructuring, and distraction. It is worth noting that no evidence yet has been reported as with college students' coping strategies during the COVID-19 pandemic.

\section{Stress Coping Styles and Hatred and Violence}

The coping strategies adopted in stressful environment may influence one's incidence of adopting negative attitude and conducting aggressive behaviors (Borders \& Liang, 2011; Yang, et al., 2021). For instance, adopting positive coping strategies, such as seeking instrumental support and using forgiveness, can alleviate one's stressor-related depression and thus decrease aggressive behaviors when facing with life stressors (Austin \& Falconier, 2012); on the other hand, young adults who use negative coping strategies, such as avoidance and substance abuse, to deal with stressors could have elevated risks of depression and internet addiction, which in turn increase one's problem behaviors (Cao \& Yang, 2018; Chou et al., 2018).

It is also worth noting that some coping strategies might influence the stigma/discrimination and aggressive behaviors through different pathways (Baillien et al., 2011). For example, as a coping strategy, rumination may mediate the correlation between people's perceived ethnic discrimination and aggressive behavior (Borders \& Liang, 2011). Moreover, Van den Brande et al. (2016) reported that problemfocused coping, reappraisal coping, and active coping can buffer the strain outcomes (e.g., bullying) of environment/ work stressors, while emotional coping and avoidant coping might increase the association.

\section{The Present Study}

Most studies on cyberbullying among college students are about cyberbullying prevalence, characteristics of cyberbullies, and the emotional and behavioral consequences for both 
victims and bullies (Gahagan et al., 2016; Gibb \& Devereux, 2014). Few studies have focused on its association with bullies' coping strategies of stressors. Moreover, still little evidence has been reported on how coping strategies correlate with stigmatizing attitude in a health crisis context. Based on the stress-coping theory (Lazarus \& Folkman, 1984), the present study aims to answer the following three research questions using a national sample of Chinese college students in the context of the national lockdown:

- Research Question 1: What are the patterns of college students' coping strategies during the COVID-19 lockdown?

- Research Question 2: What is the association between college students' coping strategy patterns and their stigmatizing attitude towards people in the COVID-19 epicenter?

- Research Question 3: What is the association between college students' coping strategy patterns and their cyberbullying behaviors towards people in the COVID19 epicenter?

\section{Methods}

\section{Participants and Procedures}

Data collection occurred between February $22^{\text {nd }}$ and February $29^{\text {th }}, 2020$, when was the peak period of COVID-19 pandemic in China and strict social distancing rules were applied nationwide. We conducted an online survey on Chinese people's coping strategies of the pandemic as well as their mental health situation. The eligibility criteria include: 1) aged 16 or above; 2) being able to provide an informed consent, and 3) living in China. Screening with criteria 1) being a current college student; and 2) not in Hubei Province (the key epidemic area in China), we included 1,673 college students into the present study.

A professional online survey platform was utilized to deliver the electronic questionnaire. The platform generated the web link and QR code for the questionnaire, and research assistants pasted the link and code on the bulletin board system (BBS) of each Chinese province's gateway website. Using the link or code, participants can access to the questionnaire and answer the questions anonymously. We also asked initial participants to diffuse the questionnaire through their social networks. All finished questionnaires were automatically sent back to and stored by the platform, which were available to be transformed into downloadable formats. Electronic informed consent was obtained from the participants before beginning the data collection. Ethical approval was obtained from the Ethics Committee for Scientific Research of the corresponding author's university.

\section{Measures}

\section{Cyberbullying Behaviors}

The cyberbullying scale devised and validated by Patchin and Hinduja (2015) was utilized to measure participants' cyberbullying behaviors. As a cyberbullying screening instrument with good criterion validity, the scale's usefulness has been demonstrated in different populations across the globe (Kazerooni et al., 2018; Palladino et al., 2017), including in China (Chen et al., 2018). It consists of nine items (e.g., "someone posted mean or hurtful comments/ picture/video about me online", "someone spread rumors about me online", "someone threatened to hurt me online"), which covers eight distinct cyberbullying behaviors and one global question on whether cyberbully others in the previous 30 days. In the present study, participants who reported one or more cyberbullying behaviors were coded as " 1 ", and those reporting no cyberbullying behaviors were coded as " 0 ".

\section{Stigmatizing Attitude}

The present study measured stigmatizing attitude by one single item, namely the participants' self-rated hatred towards people in Hubei Province. The score ranged from 0 to 10.

\section{Coping Strategy Pattern}

The present study used the Brief COPE Inventory by Carver (1997) to measure participants' coping strategies of the stressors related with the COVID-19 pandemic. It includes 14 two-item scales (e.g. "I've been turning to work or other activities to take my mind off things" and "I've been concentrating my efforts on doing something about the situation I'm in") that measure 14 conceptually differentiable coping reactions, namely acceptance, active coping, positive reframing, planning, using instrumental support, using emotional support, behavioral disengagement, self-distraction, self-blame, humor, denial, religion, venting, and substance use. Participants scored each of the 28 items from 1 to 4 with 1 being "Not true of me" and 4 being "Always true of me". That is, the value range of each subscale is from 0 to 8 .

To meet the requirement of latent class analysis, we coded the score of each subscale into a dummy variable. According to Zukel et al. (1981), categorization of the various ordinal response formats for the latent class indicators was based on an epidemiological risk factor model. This approach takes each measure and assigns a " 1 " to individuals scoring in the upper portion of the distribution and " 0 " to the remainder of 
the distribution. Following the standards set by Kosten et al. (2012), we took as close to the upper quartile as feasible assigning members of this quadrant a " 1 " for risk, namely adoption of the coping strategy.

\section{Covariates}

The present study controlled for the demographic variables of the participants, namely gender $($ male $=0$, female $=1)$, age (in years), and educational level (undergraduate $=0$, postgraduate $=1$ ). We also controlled for the participants' health and economic situation: 1) self-rated health, ranging from 1 to 5 with 1 being "very poor" and 5 being "very good"; and 2) relative socioeconomic status of the participant's current residential neighborhood in his/her prefecture, ranging from 1 to 5 with 1 being "very low" and 5 being "very high".

\section{Statistical Analysis}

The analysis process of the present study was comprised by two stages. First, the latent class analyses (LCAs) were conducted to explore the number and nature of the subtypes of the participants' coping strategy pattern based on the adoption of each of the fourteen coping strategies in the Brief COPE inventory. The advantage of this method is that it takes into account the interrelationship and combined effects between participants' coping strategies. The LCAs were tested using the Mplus statistical program with maximum likelihood parameter estimation and the EstimationMaximization algorithm (Muthén \& Muthén, 2017).

A parsimonious 2-class model was tested first, which was followed by sequentially increasing number of classes up to a 5-class model. Based on the work of Nylund, et al. (2007), four statistical fit indices for the latent class model were used in selecting the optimal number of latent classes, namely Akaike information criterion (AIC), Bayesian information criterion (BIC), the Lo-Mendell-Rubin' s adjusted likelihood ratio test (LRT), and entropy measures. Smaller observed values of AIC and BIC are better and indicate greater model parsimony. The AIC and BIC differ only with regard to the "weight" they attribute to model parsimony (Henson et al., 2007). The Lo-Mendell-Rubin' s LRT statistic is also an indicator for comparing models: a nonsignificant value $(p>0.05)$ suggests that the model with one less class should be accepted. Finally, entropy is a summary measure of classification certainty once posterior class probabilities are obtained. Entropy values can range from 0 to 1 , with higher values indicating a better classification.

In the second stage, the coping strategy class membership was used as the independent variable. It was first put into a hierarchical regression model, with stigmatizing attitude as dependent variable; then it was included in a logistic regression model, with cyberbullying behavior as dependent variable. Both models controlled for the demographic variables. The aim was to examine whether coping strategies of the pandemic were associated with college students' stigmatizing attitude and cyberbullying behaviors towards people from the COVID-19 epicenter, namely Hubei Province of China. All the data analyses were conducted using MPLUS 8.0 .

\section{Results}

\section{Descriptive Statistics}

Table 1 presents the descriptive statistics of the research variables. Among the participants, 1,083 (64.7\%) were females and 1,318 (78.8\%) were undergraduate students; their age ranged from 17 to $40(\mathrm{M}=21.42, \mathrm{SD}=2.72)$. The average score for self-rated health, neighborhood socioeconomic status, and cyberbullying behaviors was 4.25, 3.02, and 1.32, respectively. Moreover, the adoption rate of each of the fourteen coping strategies was between $25.9 \%$ (religion) and $57.1 \%$ (planning).

Table 1 Descriptive statistics for the research variables $(N=1,673)$

\begin{tabular}{ll}
\hline & $M(S D) / N(\%)$ \\
\hline Gender & \\
$\quad$ Male & $590(35.3 \%)$ \\
$\quad$ Female & $1,083(64.7 \%)$ \\
Age (17-40 years) & $21.42(2.72)$ \\
Educational level & \\
$\quad$ Undergraduates & $1,318(78.8 \%)$ \\
$\quad$ Postgraduates & $355(21.2 \%)$ \\
Self-rated health (1-5) & $4.25(.75)$ \\
Neighborhood SES (1-5) & $3.02(.97)$ \\
Stigmatizing attitude (0-10) & $2.48(2.60)$ \\
Acceptance & $697(41.7 \%)$ \\
Active coping & $647(38.7 \%)$ \\
Positive reframing & $584(34.9 \%)$ \\
Planning & $956(57.1 \%)$ \\
Using instrumental support & $701(41.9 \%)$ \\
Using emotional support & $763(45.6 \%)$ \\
Disengagement & $518(31.0 \%)$ \\
Self-distraction & $619(37.0 \%)$ \\
Self-blame & $665(39.7 \%)$ \\
Humor & $439(26.2 \%)$ \\
Denial & $546(32.6 \%)$ \\
Religion & $433(25.9 \%)$ \\
Venting & $747(44.7 \%)$ \\
Wibstance use & $435(26.0 \%)$ \\
\hline & $172(10.3 \%)$ \\
\hline
\end{tabular}


Table 2 Fit indices for the latent class analysis of problem behavior patterns

\begin{tabular}{lllll}
\hline Model & AIC & BIC & $p$ for LRT & Entropy \\
\hline 2 classes & 27,568 & 27,725 & .000 & .903 \\
3 classes & 26,025 & 26,264 & .000 & .836 \\
4 classes & 25,612 & 25,932 & .001 & .803 \\
5 classes & 25,302 & 25,703 & .587 & .801 \\
\hline
\end{tabular}

Table 3 Distribution of the 14 coping strategies in the four latent classes $(N=1,673)$

\begin{tabular}{lllll}
\hline & Class 1 & Class 2 & Class 3 & Class 4 \\
& Frequencies (within class \%) & \\
\hline Disengagement & $95.50 \%$ & $22.90 \%$ & $22.80 \%$ & $5.60 \%$ \\
Denial & $95.10 \%$ & $21.80 \%$ & $30.50 \%$ & $4.10 \%$ \\
Humor & $70.10 \%$ & $8.30 \%$ & $27.10 \%$ & $19.00 \%$ \\
Religion & $80.20 \%$ & $9.00 \%$ & $25.70 \%$ & $10.20 \%$ \\
Self-blame & $87.30 \%$ & $19.20 \%$ & $47.60 \%$ & $23.10 \%$ \\
Substance use & $93.70 \%$ & $18.60 \%$ & $13.20 \%$ & $4.10 \%$ \\
Venting & $89.20 \%$ & $15.50 \%$ & $75.20 \%$ & $9.40 \%$ \\
Use of emotional support & $72.40 \%$ & $12.70 \%$ & $83.10 \%$ & $19.60 \%$ \\
Use of instrumental support & $71.60 \%$ & $10.10 \%$ & $82.50 \%$ & $7.00 \%$ \\
Acceptance & $11.60 \%$ & $17.50 \%$ & $63.90 \%$ & $69.60 \%$ \\
Active coping & $9.70 \%$ & $7.90 \%$ & $59.70 \%$ & $78.10 \%$ \\
Planning & $75.00 \%$ & $17.00 \%$ & $82.00 \%$ & $69.00 \%$ \\
Positive reframing & $7.80 \%$ & $3.70 \%$ & $57.40 \%$ & $71.30 \%$ \\
Self-distraction & $10.10 \%$ & $14.90 \%$ & $62.20 \%$ & $54.70 \%$ \\
\hline
\end{tabular}

$N=268$ for Class $1 ; N=542$ for Class $2 ; N=521$ for Class $3 ; N=342$ for Class 4

\section{Results of the Latent Class Analyses}

The LCAs demonstrated the clustered nature of the participants' coping strategies of the COVID-19 pandemic. Table 2 reports the results of the latent class analysis model testing procedure using the fourteen latent class indicators of coping strategy. Models were tested from the 2-class to a 5 -class model. Based on the standards stated in the methods section, the four-class model showed the best model fit: the AIC and BIC were lower than the three-class model, the LRT indicated that the five-class model was not significantly better than the four-class model (and so the four-class solution should be preferred on the basis of parsimony) and the entropy value was acceptable.

Table 3 reported the endorsement rate of each coping strategy under the four-class model. As for the Class 1, participants belonging to this class were most likely to use disengagement, denial, humor, religion, self-blame, and venting to cope with the COVID-19 related stress, which belong to emotion-focused strategies. Therefore, this class was labeled as "emotional coping group", and it included 268 participants. Participants belonging to Class 2 ranked bottom in humor, religion, use of emotional support, active coping, planning, and positive reframing, and they ranked second to last in use of instrumental support. That is, they were not likely to use positive coping or seek supports. Meanwhile, the percentages of using other coping strategies for this class were also relatively low. Therefore, this class, containing 542 participants, was labelled as "inactive coping group".

Participants in Class 3 were found most likely to seek emotional and instrumental support. Meanwhile, they were most likely or second most likely to adopt positive coping strategies, including planning, self-distraction, acceptance, active coping, and positive reframing. Therefore, this class was labelled as "support-seeking and positive coping group", and it contained 521 participants. Finally, participants in Class 4 were most likely to use positive coping strategies, including acceptance, active coping, and positive reframing, while the least likely to adopt avoidant, negative, or reliant coping behaviors, including disengagement, denial, substance use, and venting. Meanwhile, they were not willing to seek supports: ranking bottom in the use of instrumental support and second to last in the use of emotional support. Considering these characteristics, we labelled this class as "independent and positive coping group", and it contained 342 participants. It is worth noting that this defining process stemmed from a comparative perspective, and all four groups of participants adopted coping strategies of different types and degree in the pandemic.

\section{Results of the Regression Analyses}

The proposed independent variable for the regression analyses, namely coping strategy patterns, had four categories, which were used to compute three dummy variables comparing the inactive coping group (Class 2), the support-seeking and positive coping group (Class 3 ), and the independent and positive coping group (Class 4 ) with the emotional coping group (Class 1). That is, the Class 1 was the reference group. Results of the hierarchical regression analysis and the logistic regression analysis were reported in Tables 4 and 5, respectively. Table 4 reveals the association between coping strategies and stigmatizing attitude towards people in Hubei Province. Compared with Class 1, the stigmatizing attitude of Class $2(b=-1.18, p<0.001)$, Class $3(b=-1.40$, $p<0.001)$, and Class $4(b=-1.80, p<0.001)$ was significantly lower, controlling for the demographic variables. It is also reported that being older $(p<0.01)$, undergraduate student $(p<0.05)$, and living in better neighborhood $(p<0.05)$ were associated with higher stigmatizing attitude.

Table 5 reports the results of the logistic regression model, with cyberbullying behaviors towards people in Hubei Province as the dependent variable. Compared with 
Table 4 Hierarchical regression results for the effects of coping strategy patterns $(N=1,673)$

\begin{tabular}{lll}
\hline & Model I & Model II \\
\hline Constant & $3.32(.79)^{* * *}$ & $3.87(.79)^{* * *}$ \\
Gender (ref: male) & $-.29(.13)^{*}$ & $-.11(.13)$ \\
Age & $.09(.03)^{* *}$ & $.10(.03)^{* *}$ \\
Education (ref: undergraduate) & $-.48(.21)^{*}$ & $-.49(.21)^{*}$ \\
Self-rated health & $-.17(.08)^{*}$ & $-.11(.08)$ \\
Neighborhood SES & $.16(.07)^{*}$ & $.15(.06)^{*}$ \\
Class 2 vs. Class 1 & & $-1.18(.21)^{* * *}$ \\
Class 3 vs. Class 1 & & $-1.40(.19)^{* * *}$ \\
Class 4 vs. Class 1 & & $-1.80(.21)^{* * *}$ \\
$R^{2}$ adjusted & $.010^{* * *}$ & $.054^{* * *}$ \\
\hline
\end{tabular}

The dependent variable is stigmatizing attitude. The reference group is Class 1 (i.e., the emotional coping group). ${ }^{*} p<.05 ;{ }^{* *} p<.01$; ${ }^{* * *} p<.001$

Table 5 Logistic regression results for the effects of coping strategy patterns $(N=1,673)$

\begin{tabular}{lll}
\hline & Model I & Model II \\
\hline Constant & .33 & .52 \\
Gender (ref: male) & $.33[.24, .46]^{* * *}$ & $.40[.28, .55]^{* * *}$ \\
Age & $.97[.89,1.05]$ & $.98[.90,1.06]$ \\
Education (ref: undergradu- & $1.30[.76,2.22]$ & $1.26[.72,2.21]$ \\
$\quad$ ate) & $.89[.72,1.10]$ & $.95[.76,1.18]$ \\
Self-rated health & $1.29[1.10,1.53]^{* *}$ & $1.28[1.08,1.52]^{* *}$ \\
Neighborhood SES & & $.29[.20, .44]^{* * *}$ \\
Class 2 vs. Class 1 & & $.28[.18, .43]^{* * *}$ \\
Class 3 vs. Class 1 & & $.13[.07, .24]^{* * *}$ \\
Class 4 vs. Class 1 & $.064^{* * *}$ & $.142^{* * *}$ \\
$R^{2}$ (Nagelkerke) & & \\
\hline
\end{tabular}

The dependent variable is cyberbullying behaviors, a dummy variable. The reference group is Class 4 (i.e., the independent and positive coping group). ${ }^{*} p<.05 ;{ }^{* *} p<.01 ;{ }^{* * *} p<.001$

Class 1, the likelihood of cyberbullying behaviors was $71 \%$ lower for Class 2 (Odds ratio $=0.29, p<0.001), 72 \%$ lower for Class 3 (Odds ratio $=0.28, p<0.001$ ), and $87 \%$ lower for Class 4 (Odds ratio $=0.13, p<0.001$ ), controlling for the demographics of the college students. It is also reported that being male $(p<0.001)$ and living in better neighborhood $(p<0.01)$ were associated with higher likelihood of cyberbullying.

\section{Discussion}

By investigating a national sample of Chinese college students, the present study provides the following answers to the three research questions. First, we found that the coping strategies of the college students during the pandemic were clustered. Four latent subgroups were identified among the participants based on type and comparative adoption rate of their coping strategies, namely the emotional coping group, the inactive coping group, the support-seeking and positive coping group, and the independent and positive coping group. Second, the stigmatizing attitude towards people in Hubei Province, the place where the first COVID-19 case was reported and recognized as China's epicenter of the pandemic, was significantly different between latent subgroups. Third, the cyberbullying behaviors towards people in Hubei Province, was significantly different between latent subgroups. It is reported that participants adopting emotional coping had highest stigmatizing attitude and were most likely to have cyberbullying behaviors.

The results as with the clustered nature of coping strategy are in line with the previous studies that reported people's stress coping strategies showed a multiple-factor structure. However, different from Endler et al. (1998)'s three-factor model and Doron et al. (2014)'s five-factor model, we revealed a four-factor coping strategy pattern among college students. It is noted that the appropriateness and generalizability of coping strategy typologies are still being debated (Skinner \& Zimmer-Gembeck, 2007). Scholars have proposed many alternative categorizations such as through the addition of avoidant coping (Weiss et al., 2019) or dysfunctional coping (Okech et al., 2018). It is argued that type of stressors, resource availability, and stage of recovery should be considered to determine which range of strategies and level of specificity allows for the most efficient assessment of people's use of coping strategies (Doron et al., 2014).

The findings as with the participants' stigmatizing attitude and cyberbullying behaviors towards people in pandemic epicenter as well as their associations with coping strategies are in line with the past literature. According to Puhl and Heuer (2009), stigmatizations can lead societies to form prejudices, frame negative stereotyping, and encourage aggressions towards vulnerable people. These associations are strengthened more than ever in the digital age, when young adults become less cautious in expressing their opinions or prejudices in social media, showing little regard on the impact their views may have at the receiving end (Lim, 2017; Yang \& Zhang, 2018). Since internet and social media have opened spaces for conducting different discourses, it becomes a challenge to maintain a complex balance between freedom of expression and the defense of human dignity. For example, Wanniarachchi et al. (2020) noted that when large groups of people post similar biased expressions over a publicly shared medium, the onslaught of negative content can tilt our perceptions on what is considered to be acceptable and what is not acceptable. Moreover, the anonymity brought about by virtuality on the internet makes restraints on voicing one's opinions in open forum void. 
Furthermore, past literature also supports the significant role of coping strategies in buffering or exacerbating hatred and violence (Mishna et al., 2020). The present study gives a more detailed picture by revealing that stigmatizing attitude and cyberbullying behaviors were most likely to happen among the participants using emotional coping while the least likely among the independent and positive coping group. According to Völlink et al. (2013), using emotional coping strategies is associated with negative personality characteristics and does not deal with stressors directly, thus it could predict behavioral problems such as agitation and aggression. On the other hand, coping with stressors independently and positively indicates resourcefulness and positive personalities (e.g., optimistic and persistent) that buffer behavioral consequences of stressors (Chao, 2011).

The findings from the present study should be interpreted with the following caveats. First, the cross-sectional design of this study precluded the establishment of causal links between coping strategies, stigmatizing attitude, and cyberbullying behaviors. Future longitudinal research is required to assess change in coping strategy pattern and its influence on stigmatizing attitude and cyberbullying behaviors. Second, although the present study had a nationwide scale, it was based on an online survey which precluded college students who had no access to smart phone or internet during the pandemic. Also, we recruited participants mainly through bulletin board system, which is relatively less used by current Chinese college students. Therefore, it should be cautioned to generalize the findings to the whole Chinese college students. Third, stigmatizing attitude was measured by one global question in this analysis, which might not be accurate enough. Future studies shall apply a multi-dimensional scale to test its prevalence in populations under the background of public health crisis. Fourth, the four-factor structure of coping strategies among Chinese college students might not be applied to their counterparts in other countries, due to the differences in culture, stage of crisis, and government policies on COVID-19. Future studies may compare the factor structure of coping strategies applied by college students in different countries and delve into the factors that contribute to the difference.

\section{Conclusions}

The present study demonstrates the associations between coping strategy patterns and stigmatizing attitude and cyberbullying behaviors among college students during the COVID-19 lockdown. Moreover, it enhances the understanding of college students' coping strategies of the pandemic. The findings regarding the prevalence of stigmatizing attitude and cyberbullying behaviors towards people in Hubei Province, as well as their correlation with coping strategies provide empirical supports for combating the secondary disasters of the pandemic, namely stigma and cyberbullying, that not only harm our mental health but also tear our world apart. The stigmatization experiences of Hubei residents in China could be identical with those of Asians or people from major epidemic areas in other parts of the world (World Health Organization, 2020). It is suggested that governments, mental health services, and mass media should work together to encourage college students to avoid emotional coping style and to adopt more positive coping strategies that deal directly with the source of the stress, in order to alleviate the behavioral and emotional consequences of the COVID-19 pandemic.

Data Availability The datasets generated during and/or analyzed during the current study are not publicly available due to the requirement of the sponsor but are available from the corresponding author on reasonable request.

\section{Declarations}

Conflict of Interest None.

\section{References}

Alsawalqa, R. O. (2021). Cyberbullying, social stigma, and self-esteem: The impact of COVID-19 on students from East and Southeast Asia at the University of Jordan. Heliyon, 7(4), e06711. https:// doi.org/10.1016/j.heliyon.2021.e06711

Altena, E., Baglioni, C., Espie, C. A., Ellis, J., Gavriloff, D., Holzinger, B., ... Riemann, D. (2020). Dealing with sleep problems during home confinement due to the COVID-19 outbreak: Practical recommendations from a task force of the European CBT-I Academy. Journal of Sleep Research, 29(4), e13052.https://doi.org/10.1111/ jsr. 13052

Austin, J. L., \& Falconier, M. K. (2012). Spirituality and common dyadic coping. Journal of Family Issues, 34(3), 323-346. https:// doi.org/10.1177/0192513x12452252

Baillien, E., De Cuyper, N., \& De Witte, H. (2011). Job autonomy and workload as antecedents of workplace bullying: A two-wave test of Karasek's Job Demand Control Model for targets and perpetrators. Journal of Occupational and Organizational Psychology, 84(1), 191-208. https://doi.org/10.1348/096317910x508371

Bodenmann, G., Meuwly, N., Bradbury, T. N., Gmelch, S., \& Ledermann, T. (2010). Stress, anger, and verbal aggression in intimate relationships: Moderating effects of individual and dyadic coping. Journal of Social and Personal Relationships, 27(3), 408-424. https://doi.org/10.1177/0265407510361616

Borders, A., \& Liang, C. T. H. (2011). Rumination partially mediates the associations between perceived ethnic discrimination, emotional distress, and aggression. Cultural Diversity and Ethnic Minority Psychology, 17(2), 125-133. https://doi.org/10.1037/ a0023357

Cao, Y., \& Yang, F. (2018). Self-efficacy and problem behaviors of school bully victims: Evidence from rural China. Journal of Child and Family Studies, 27(10), 3241-3249. https://doi.org/10.1007/ s10826-018-1150-0 
Carver, C. S. (1997). You want to measure coping but your protocol' too long: Consider the brief cope. International Journal of Behavioral Medicine, 4(1), 92. https://doi.org/10.1207/s1532 7558ijbm0401_6

Chao, R.C.-L. (2011). Managing stress and maintaining well-being: Social support, problem-focused coping, and avoidant coping. Journal of Counseling \& Development, 89(3), 338-348. https:// doi.org/10.1002/j.1556-6678.2011.tb00098.x

Chen, Q., Lo, C. K. M., Zhu, Y., Cheung, A., Chan, K. L., \& Ip, P. (2018). Family poly-victimization and cyberbullying among adolescents in a Chinese school sample. Child Abuse \& Neglect, 77, 180-187. https://doi.org/10.1016/j.chiabu.2018.01.015

Chiolero, A. (2020). Covid-19: A digital epidemic. BMJ, 368, m764. https://doi.org/10.1136/bmj.m764

Chou, W. P., Yen, C. F., \& Liu, T. L. (2018). Predicting effects of psychological inflexibility/experiential avoidance and stress coping strategies for internet addiction, significant depression, and suicidality in college students: A prospective study. International Journal of Environmental Research and Public Health, 15(4), 788. https://doi.org/10.3390/ijerph15040788

DiClemente, C. M., \& Richards, M. H. (2019). Community violence in early adolescence: Assessing coping strategies for reducing delinquency and aggression. Journal of Clinical Child \& Adolescent Psychology, 1-14.https://doi.org/10.1080/15374416.2019. 1650365

Doron, J., Trouillet, R., Gana, K., Boiche, J., Neveu, D., \& Ninot, G. (2014). Examination of the hierarchical structure of the brief COPE in a French sample: Empirical and theoretical convergences. Journal of Personality Assessment, 96(5), 567-575. https://doi.org/10.1080/00223891.2014.886255

Endler, N. S., Parker, J. D. A., \& Summerfeldt, L. J. (1998). Coping with health problems: Developing a reliable and valid multidimensional measure. Psychological Assessment, 10(3), 195-205. https://doi.org/10.1037/1040-3590.10.3.195

Fluharty, M., \& Fancourt, D. (2021). How have people been coping during the COVID-19 pandemic? Patterns and predictors of coping strategies amongst 26,016 UK adults. BMC Psychology, 9, 107. https://doi.org/10.1186/s40359-021-00603-9

Gahagan, K., Vaterlaus, J. M., \& Frost, L. R. (2016). College student cyberbullying on social networking sites: Conceptualization, prevalence, and perceived bystander responsibility. Computers in Human Behavior, 55, 1097-1105. https://doi.org/10.1016/j.chb. 2015.11.019

Garaigordobil, M., \& Larrain, E. (2020). Bullying and cyberbullying in LGBT adolescents: Prevalence and effects on mental health. Comunicar, 28(62), 79-90. https://doi.org/10.3916/c62-2020-07

Gibb, Z. G., \& Devereux, P. G. (2014). Who does that anyway? Predictors and personality correlates of cyberbullying in college. Computers in Human Behavior, 38, 8-16. https://doi.org/10.1016/j. chb.2014.05.009

Goh, B. (2020). In China's Hubei, uncertainty, pessimism and hope as life resumes. Reuters. https://www.reuters.com/article/us-healthcoronavirus-china-hubei-idUSKBN21E0CT

Henson, J. M., Reise, S. P., \& Kim, K. H. (2007). Detecting mixtures from structural model differences using latent variable mixture modeling: A comparison of relative model fit statistics. Structural Equation Modeling: A Multidisciplinary Journal, 14(2), 202-226. https://doi.org/10.1080/10705510709336744

Holmes, E. A., O'Connor, R. C., Perry, V. H., Tracey, I., Wessely, S., Arseneault, L., ... Bullmore, E. (2020). Multidisciplinary research priorities for the COVID-19 pandemic: a call for action for mental health science. The Lancet Psychiatry, 7(6), 547-560.https://doi. org/10.1016/s2215-0366(20)30168-1

Kazerooni, F., Taylor, S. H., Bazarova, N. N., \& Whitlock, J. (2018). Cyberbullying bystander intervention: The number of offenders and retweeting predict likelihood of helping a cyberbullying victim. Journal of Computer-Mediated Communication, 23(3), 146-162. https://doi.org/10.1093/jcmc/zmy005

Kim, K. Y., \& Choi, J. S. (2021). Cyberbullying, student nurses' ethical awareness and the Covid-19 pandemic. Nursing Ethics, 28(7-8), 1258-1268. https://doi.org/10.1177/0969733021 1010280

Kosten, P. A., Scheier, L. M., \& Grenard, J. L. (2012). Latent class analysis of peer conformity. Youth \& Society, 45(4), 565-590. https://doi.org/10.1177/0044118x12454307

Lauckner, C., Truszczynski, N., Lambert, D., Kottamasu, V., Meherally, S., Schipani-McLaughlin, A. M., ... Hansen, N. (2019). "Catfishing," cyberbullying, and coercion: An exploration of the risks associated with dating app use among rural sexual minority males. Journal of Gay \& Lesbian Mental Health, 23(3), 289-306.https://doi.org/10.1080/19359705.2019.1587729

Lazarus, R. S., \& Folkman, S. (1984). Stress, appraisal, and coping: Springer publishing company.

Lim, M. (2017). Freedom to hate: Social media, algorithmic enclaves, and the rise of tribal nationalism in Indonesia. Critical Asian Studies, 49(3), 411-427. https://doi.org/10.1080/14672715.2017. 1341188

McCauley, M., Minsky, S., \& Viswanath, K. (2013). The H1N1 pandemic: Media frames, stigmatization and coping. BMC Public Health, 13(1), 1116. https://doi.org/10.1186/1471-2458-13-1116

Mishna, F., Sanders, J. E., McNeil, S., Fearing, G., \& Kalenteridis, K. (2020). "If somebody is different": A critical analysis of parent, teacher and student perspectives on bullying and cyberbullying. Children and Youth Services Review. https://doi.org/10.1016/j. childyouth.2020.105366

Muthén, L., \& Muthén, B. (2017). Mplus user's guide (8th ed.). http:// www.statmodel.com/download/usersguide/MplusUserGuideV er_8.pdf

Nylund, K. L., Asparouhov, T., \& Muthén, B. O. (2007). Deciding on the number of classes in latent class analysis and growth mixture modeling: A Monte Carlo simulation study. Structural Equation Modeling, 14(4), 535-569. https://doi.org/10.1080/1070551070 1575396

Okech, D., Hansen, N., Howard, W., Anarfi, J. K., \& Burns, A. C. (2018). Social support, dysfunctional coping, and community reintegration as predictors of PTSD among human trafficking survivors. Behavioral Medicine, 44(3), 209-218. https://doi.org/ 10.1080/08964289.2018.1432553

Palladino, B. E., Menesini, E., Nocentini, A., Luik, P., Naruskov, K., Ucanok, Z., ... Scheithauer, H. (2017). Perceived severity of cyberbullying: Differences and similarities across four countries. Frontiers in Psychology, 8, 1524.https://doi.org/10.3389/fpsyg. 2017.01524

Patchin, J. W., \& Hinduja, S. (2015). Measuring cyberbullying: Implications for research. Aggression and Violent Behavior, 23, 69-74. https://doi.org/10.1016/j.avb.2015.05.013

Puhl, R. M., \& Heuer, C. A. (2009). The stigma of obesity: A review and update. Obesity (silver Spring), 17(5), 941-964. https://doi. org/10.1038/oby.2008.636

Qiu, J., Shen, B., Zhao, M., Wang, Z., Xie, B., \& Xu, Y. (2020). A nationwide survey of psychological distress among Chinese people in the COVID-19 epidemic: Implications and policy recommendations. General Psychiatry, 33(2), e100213. https://doi.org/ 10.1136/gpsych-2020-100213

Skinner, E. A., \& Zimmer-Gembeck, M. J. (2007). The development of coping. Annual Review of Psychology, 58, 119-144. https://doi. org/10.1146/annurev.psych.58.110405.085705

Taylor, S. E., \& Stanton, A. L. (2007). Coping resources, coping processes, and mental health. Annual Review of Clinical Psychology, 3, 377-401. https://doi.org/10.1146/annurev.clinpsy.3.022806. 091520 
Van den Brande, W., Baillien, E., De Witte, H., Vander Elst, T., \& Godderis, L. (2016). The role of work stressors, coping strategies and coping resources in the process of workplace bullying: A systematic review and development of a comprehensive model. Aggression and Violent Behavior, 29, 61-71. https://doi.org/10. 1016/j.avb.2016.06.004

Völlink, T., Bolman, C., Eppingbroek, A., \& Dehue, F. (2013). Emotion-focused coping worsens depressive feelings and health complaints in cyberbullied children. Journal of Criminology. https:// doi.org/10.1155/2013/416976

Wanniarachchi, V. U., Mathrani, A., Susnjak, T., \& Scogings, C. (2020). A systematic literature review: What is the current stance towards weight stigmatization in social media platforms? International Journal of Human-Computer Studies, 135, 102371. https:// doi.org/10.1016/j.ijhcs.2019.102371

Weiss, N. H., Risi, M. M., Sullivan, T. P., Armeli, S., \& Tennen, H. (2019). Post-traumatic stress disorder symptom severity attenuates bi-directional associations between negative affect and avoidant coping: A daily diary study. Journal of Affective Disorders, 259, 73-81. https://doi.org/10.1016/j.jad.2019.08.015

Wong, A. H., Roppolo, L. P., Chang, B. P., Yonkers, K. A., Wilson, M. P., Powsner, S., \& Rozel, J. S. (2020). Management of agitation during the COVID-19 pandemic. Western Journal of Emergency Medicine: Integrating Emergency Care with Population Health, 21(4), 795-800. https://doi.org/10.5811/westjem.2020.5.47789

World Health Organization. (2020). Mental health and psychosocial considerations during the COVID-19 outbreak. https://apps. who. int/iris/handle/10665/331490
Yang, F. (2021). Coping strategies, cyberbullying behaviors, and depression among Chinese netizens during the COVID-19 pandemic: A web-based nationwide survey. Journal of Affective Disorders, 281, 138-144. https://doi.org/10.1016/j.jad.2020.12.023

Yang, F., \& Huang, Z. (2021). Health communication and trust in institutions during the COVID-19 lockdown in China's urban communities. Urban Governance, 1, 17-24. https://doi.org/10.1016/j. ugj.2021.10.001

Yang, F., Shen, Y., \& Nehring, D. (2021). Maltreatment and depression among left-behind adolescents in rural China: The moderating roles of food security and depression literacy. Child Abuse and Neglect, 114, 104976. https://doi.org/10.1016/j.chiabu.2021. 104976

Yang, F., \& Zhang, L. (2018). Problem behavior patterns of victims of school bullying in rural China: The role of intrapersonal and interpersonal resources. Children and Youth Services Review, 93, 315-320. https://doi.org/10.1016/j.childyouth.2018.08.007.

Zukel, W. J., Paul, O., \& Schnaper, H. W. (1981). The multiple risk factor intervention trial (MRFIT): I. Historical perspectives. Preventive Medicine, 10(4), 387-401. https://doi.org/10.1016/00917435(81)90057-8

Publisher's Note Springer Nature remains neutral with regard to jurisdictional claims in published maps and institutional affiliations. 